\title{
Trends and Outcomes of Sacral Neuromodulation: A Saudi Tertiary Care Center Experience
}

\author{
(1) Mohammad Alghafees 1 , (1) Yahya Ghazwani22, (1) Meshari Alqahtani1, (1) Rakan Aldarrab2 \\ ${ }^{1}$ King Saud bin Abdulaziz University for Health Sciences College of Medicine, Riyadh, Saudi Arabia \\ ${ }^{2}$ King Abdulaziz Medical City, Department of Urology, Riyadh, Saudi Arabia
}

\section{What's known on the subject? and What does the study add?}

The literature presents a gap when it comes to the trends and outcomes of sacral neuromodulation (SNM) globally, especially in the Middle East. This study evaluated the trends and outcomes of SNM in our patient population. The study could be an incentive for larger multicenter studies. Exploring the outcomes of SNM on a larger scale will improve the procedure.

\section{Abstract}

Objective: Sacral neuromodulation (SNM) is a validated treatment for overactive bladder syndrome and chronic non-obstructive retention. In Saudi Arabia, SNM is gaining popularity. It improves patient outcomes and eliminates the associated stigma with refractory urine retention. This study aimed to assess the trends and outcomes in patients with SNM in King Abdulaziz Medical City.

Materials and Methods: This retrospective cohort study targeted adult patients who underwent an SNM device implantation between January 1 , 2016, and January 1, 2021. Frequency and percentage were used to display the categorical variables and minimum, maximum, mean, and standard deviation for the continuous variables.

Results: Of the 28 patients, $13(46.4 \%)$ were males and 15 (53.6\%) females. The mean age was $37.14+14.62$ years. The most frequent indication was idiopathic bladder dysfunction $(28.6 \%, \mathrm{n}=8)$. The first stage success rate was $53.6 \%$, of which $42.9 \%$ had the device permanently implanted in the second stage. The overall complication rates were $66.6 \%$ and $42.84 \%$ for device change and electrode change, respectively, with the most frequent complications as device protrusion and dislocation after device change $(n=1,33.3 \%)$ and urinary tract infections after electrode change $(n=3,21.42 \%)$

Conclusion: The complication rate was similar to the literature. However, the first stage success rate was lower than the reported local and international rates. Regular documentation before and after implantation is important to gather data for future studies. Exploring the outcomes of SNM on a larger scale will improve preoperative, perioperative, and postoperative care, thereby supporting more patient satisfaction.

Keywords: Sacral neuromodulation, neurourology, bladder dysfunction

\section{Introduction}

Since its introduction in the 1990s, sacral neuromodulation (SNM) has been a beneficial treatment for chronic dysfunction of the urinary system, the bowels, and the pelvic floor. The first sacral SNM implantable systems were approved in 1997 by the Food and Drug Administration for urgency incontinence. According to the provided data by the American Board of Urology, SNM procedures constituted $76 \%$ of surgeries to rectify overactive bladder (OAB) dysfunction in 2012 (1). SNM has become a popular option for refractory $O A B$ over the last 20 years and is an effective treatment for $O A B$ and urge incontinence, resulting in a decreased number of voids, increased bladder holding capacity, normal bladder residual volume, and fewer leakage episodes. It has led to a higher quality of life, lower depression rate, and better quality of sleep (2). Globally, $>150,000$ procedures have been performed (2).

Correspondence: Mohammad Alghafees MD, King Saud bin Abdulaziz University for Health Sciences College of Medicine, Riyadh, Saudi Arabia E-mail: Alghafees687@gmail.com ORCID-ID: orcid.org/0000-0003-1136-9578 Received: 29.05.2021 Accepted: 07.10.2021

Cite this article as: Alghafees M, Ghazwani Y, Alqahtani M, Aldarrab R. Trends and Outcomes of Sacral Neuromodulation: A Saudi Tertiary Care Center Experience. J Urol Surg, 2022;9(1):25-32.

๑Copyright 2022 by the Association of Urological Surgery / Journal of Urological Surgery published by Galenos Publishing House. 
SNM is an approved and validated treatment for OAB syndrome, chronic non-obstructive retention, and chronic pelvic pain. A literature review that assessed the current evidence available about SNM reported it as a safe and effective third-line treatment. The overall success rate ranges from $43 \%$ to $85 \%$. Due to the invasive surgical technique and the presence of a permanent implant, SNM has a relatively high surgical revision rate, ranging from $9 \%$ to $33 \%$ (3). The most prevalent reported complications to include pain at the implant site (15-42\%), followed by lead migration (4-21\%), pain at the lead site (5.4$19 \%)$, leg pain (18\%), and infection (5.7-6.1\%) (3). The quality of studies related to SNM is generally low due to insufficient randomized controlled trials, and are mostly prospective observational studies with mid-term follow-up.

In Saudi Arabia, SNM is gaining popularity because it improves patient outcomes and eliminates the associated stigma with refractory urine retention (non-obstructive), $O A B$, chronic pelvic pain, chronic constipation, and fecal incontinence. This study aimed to assess the trends and outcomes of patients who received SNM at King Abdulaziz Medical City (KAMC).

\section{Materials and Methods}

This retrospective cohort study was conducted at KAMC, Riyadh, Saudi Arabia. All patients who underwent SNM implantation from January 1, 2016, to January 1, 2021, were included. Patients younger than 14 years and those with SNM implantation in a different hospital and followed-up at KAMC were excluded.

The variables, including demographic information, comorbidities, diagnosis, SNM indication, failure or success at the first and second stage of implantation, number of changes done in the time interval, and complications post-change, were extracted from the BESTCare system (ezCareTech, South Korea).

The Institutional Review Board of King Abdullah International Medical Research Center, the Ministry of National Guard-
Health Affairs, Riyadh, Kingdom of Saudi Arabia, approved the study with the approval number NRC21R/095/03. The patients' confidentiality and anonymity were ensured, as serial numbers replaced the medical record numbers. The data was accessed and used by only the research team.

\section{Statistical Analysis}

The data were entered in Microsoft Excel 2019 (Microsoft Corporation, WA, USA), and the statistical analysis was done with the Statistical Package for the Social Sciences version 23.0 (IBM Corporation, NY, USA). Frequency and percentage are used to display the categorical variables and the minimum, maximum, mean, and standard deviation to display the continuous variables.

\section{Results}

The sample size was realized as 28 patients $(n=28)$. Table 1 displays the sociodemographic profile of the sample. Almost half $(n=13,46.4 \%)$ were males and $15(53.6 \%)$ females. The minimum age was 17 years and the maximum was 73 years, with a mean of $37.14 \pm 14.62$ years.

Figure 1 displays the comorbidities occurring in the sample. More than half $(n=16,57.1 \%)$ of the patients were medically

\begin{tabular}{|l|l|l|}
\hline \multicolumn{4}{|l|}{ Table 1. Sociodemographic profile of patients (n=28) } \\
\hline Demographical characteristics & n & \% \\
\hline Gender & 13 & 46.4 \\
\hline Male & 15 & 53.6 \\
\hline Female & \multicolumn{2}{|l|}{} \\
\hline Age & 37.14 \\
\hline Mean & 14.62 \\
\hline Standard deviation & 17 \\
\hline Minimum & 73 \\
\hline Maximum & \multicolumn{2}{|l}{} \\
\hline
\end{tabular}

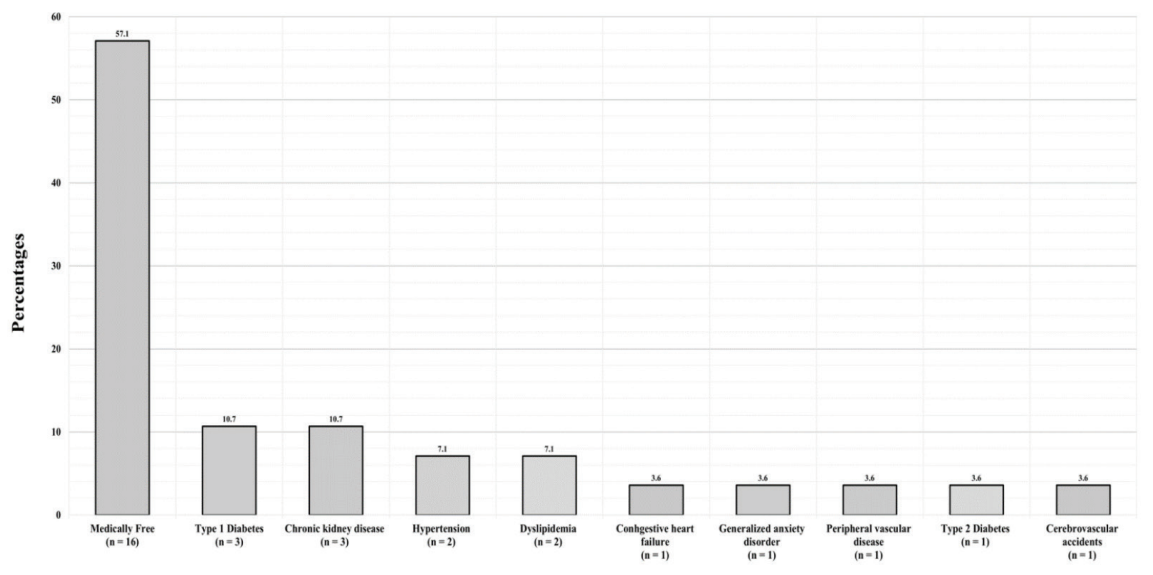

Figure 1. Presence of Co-morbidities among the participants 
free, 3 (10.7\%) had type 1 diabetes, 3 (10.7\%) had chronic kidney disease, 2 (7.1\%) had hypertension, 2 (7.1\%) had dyslipidemia, $1(3.6 \%)$ had congestive heart failure, 1 (3.6\%) had generalized anxiety disorder, 2 (3.6\%) had peripheral vascular disease, 1 (3.6\%) had type 2 diabetes, and 1 (3.6\%) had cerebrovascular accident.

Figure 2 presents the patients' diagnoses. The highest proportion $(n=8,28.6 \%)$ was idiopathic bladder dysfunction, followed by 6 $(21.4 \%)$ with neurogenic bladders due to a spinal cord injury, 4 $(14.3 \%)$ with $O A B, 2$ (7.1\%) with urinary incontinence, 2 (7.1\%) with chronic urinary retention, 2 (7.1\%) with spina bifida, $2(7.1 \%)$ with dysfunctional voiding, 1 (3.6\%) with Fowler syndrome, and 1 (3.6\%) with chronic interstitial cystitis.

Table 2 demonstrates the trials done with the patients and the outcome. More than half $(n=15,53.6 \%)$ had a successful first trial. The second stage trial was not performed in 16 (57.1\%) and was successful in 12 (42.9\%) patients.

Table 3 illustrates the device change history. A small proportion $(n=3,10.7 \%)$ of the sample had one device change, with the majority $(n=25,89.3 \%)$ never requiring a device change. The device change for the first patient was performed after 28 months and was complicated by the device protruding from the incision site, with minimal discharge. The second patient was after 42 months, complicated by the bulging of the device

\begin{tabular}{|l|l|l|}
\hline \multicolumn{3}{|l|}{ Table 2. Trials and their outcome (n=28) } \\
\hline Question & $\mathbf{n}$ & $\%$ \\
\hline Trial at first stage & 15 & 53.6 \\
\hline Succeeded & 13 & 46.4 \\
\hline Failed & 16 & 57.1 \\
\hline Trial at second stage & 12 & 42.9 \\
\hline Not done & 12 \\
\hline Succeeded
\end{tabular}

with pain around the device are and mild tenderness. The third patient interval was not recorded; however, no complication was associated with this device change.

Table 4 displays the battery change history. The majority $(n=26$, $92.8 \%)$ did not require a battery change, 1 (3.6\%) patient had a battery change once and another 1 (3.6\%) twice. The single battery change of the first patient occurred at 60 months and was not associated with complications. The first of the two battery changes in another patient was done at 24 months, without complications, and the second was done after 24 months, without complications.

Table 5 shows the electrode change/readjustment history. The majority of the sample $(n=25,89.2 \%)$ never had an electrode change/readjustment; however, 1 (3.6\%) had it twice, 1 (3.6\%) three times, and another $1(3.6 \%) 9$ times.

\begin{tabular}{|c|c|c|}
\hline Question & $\mathbf{n}$ & $\%$ \\
\hline \multicolumn{3}{|c|}{ How many device changes were done? } \\
\hline None & 25 & 89.3 \\
\hline 1 & 3 & 10.7 \\
\hline \multicolumn{3}{|c|}{ Patient 1 with device changes } \\
\hline Time Interval & \multicolumn{2}{|c|}{28 months } \\
\hline Complication incidence & \multicolumn{2}{|c|}{$\begin{array}{l}\text { Device protrusion from incision } \\
\text { site with minimal discharge }\end{array}$} \\
\hline \multicolumn{3}{|c|}{ Patient 2 with device changes } \\
\hline Time Interval & \multicolumn{2}{|c|}{42 months } \\
\hline Complication incidence & \multicolumn{2}{|c|}{$\begin{array}{l}\text { Bulging of the device, with pain } \\
\text { around the device and mild } \\
\text { tenderness }\end{array}$} \\
\hline \multicolumn{3}{|c|}{ Patient 3 with device changes } \\
\hline Time Interval & \multicolumn{2}{|c|}{ Not documented } \\
\hline Complication incidence & \multicolumn{2}{|c|}{ None } \\
\hline
\end{tabular}

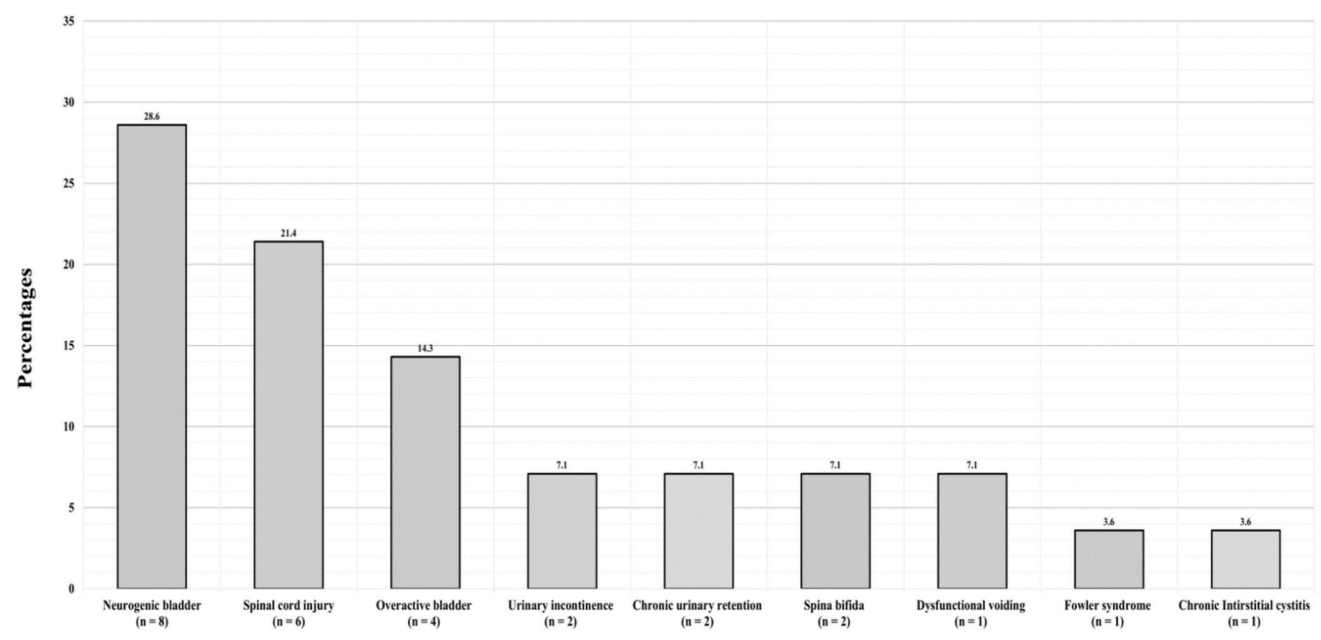

Figure 2. Diagnosis of the patients 
The patient who required two electrode changes/readjustments had the first due to a history of trauma at the site of the device, which after the patient complained of an interrupted stream, at 3 months, without complications. The second was due to device erosion and malfunction at 9 months, without complications.

The second patient who required three electrode changes/ readjustments had the first due to device displacement at 1.5 months, the second due to electrode displacement, causing a feeling of hesitancy and not feeling the vibrations at 21 months, and the third due to electrode displacement at 25 months, all without complications.

The third patient who required nine electrode changes/ readjustments had the first due to pain at the site of the device at 12 months, with urinary incontinence; the second due to urinary incontinence at 4 months, with urinary tract infection; the third due to device erosion after 2 months, with urinary tract infection; the fourth due to a superficially palpable device after 4 months, with pain; the fifth due to electrode displacement at 7 months, with exposed wire from the incision site and minimal discharge; the sixth due to an exposed wire after 1.5 months,

\begin{tabular}{|l|l|l|}
\hline Table 4. Battery change history (n=28) & $\mathbf{n}$ & $\%$ \\
\hline Question & 0 & 92.9 \\
\hline How many battery changes were done? & 1 & 3.6 \\
\hline None & 1 & 3.6 \\
\hline 1 & \multicolumn{3}{|l|}{} \\
\hline 2 & 60 months \\
\hline Patient 1 with battery change once & None \\
\hline Time interval & \multicolumn{3}{|l|}{} \\
\hline Complication incidence & 24 months \\
\hline Patient 2 with device changes twice & None \\
\hline Time interval in first battery change & \multicolumn{2}{|l|}{24 months } \\
\hline Complication incidence in first battery change \\
\hline Time interval in second battery change & \multicolumn{2}{|l|}{ None } \\
\hline Complication incidence in second battery change & \multicolumn{2}{|l|}{} \\
\hline
\end{tabular}

without complications; the seventh due to lower leg numbness triggered by turning the device on after 12 months, without complications; the eighth due to the protrusion of the device after 11 months, without complication; and the ninth due to the protrusion of the device at 1.5 months, with urinary tract infection.

Figure 3 displays the rate of postoperative revisits. The revisits were due to a device change in $3(10.7 \%)$ patients, battery change in $3(10.7 \%)$, and electrode change/readjustment in 14 (50\%).

Figure 4 demonstrates the complication rate in patients who had a device change. The overall rate of complications was 2 (66.6\%). Device protrusion from the incision site, with minimal discharge, occurred in 1(33.3\%) patient and the second (33.3\%) due to devise bulging, with pain around the device and mild tenderness.

Figure 5 illustrates the complication rate in the group with an electrode change/readjustment. The overall rate of complications was $6(42.84 \%)$, of which $3(21.42 \%)$ had urinary tract infection, $1(7.14 \%)$ urinary incontinence, 1 (7.14\%) pain, and 1 (7.14\%) exposed wire from the incision site, with minimal discharge.

\section{Discussion}

The current study presents an overview of the experience and follow-up in patients with SNM in Saudi attending KAMC. The mechanism of action of SNM and the clinical outcome of the therapy remained controversial in the literature; however, the results support the persistent clinical advantage of SNM, as reported in many studies (4-7). Many reports vary in terms of an association between the demographic characteristics, such as age and gender, and SNM outcome (4-7). In terms of gender, studies in the United States of America (USA) and China reported similar outcomes in patients of both genders $(5,6)$. However, a study done in Iraq reported a difference in the SNM outcome, with females having better outcomes than males. This finding

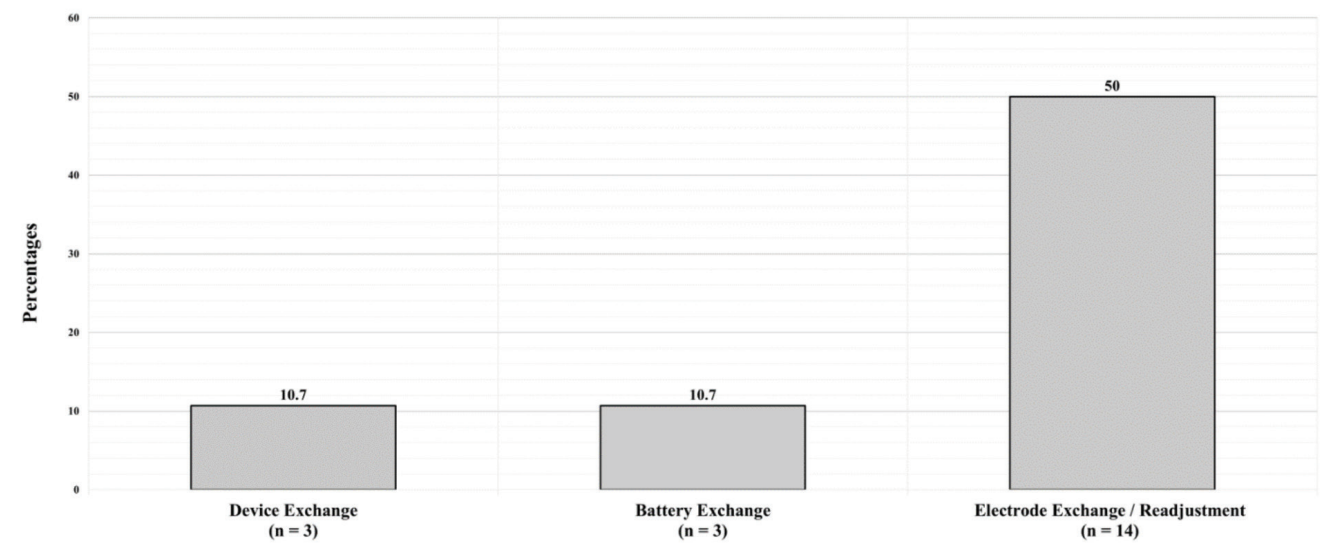

Figure 3. Overall rate of post-op revisits for device exchange, battery exchange and electrode exchange/readjustment 
could be attributed to the different indications for the SNM in the two gender groups (8). The current study revealed that gender distribution was equally proportioned, with 13 (46.4\%) males and $15(53.6 \%)$ females, contrary to what is reported in the literature. In Kuwait, the experience of the only center performing SNM reported a major female predominance (81\%) (9). Similarly, a study in the Netherlands reported a female predominance of $83 \%$ in the sample (7). These differences could influence the success rate in both genders. In terms of age, the study's sample had a mean age of $37.14 \pm 14.62$ years, consistent with the samples reported in the literature $(7,9,10)$.

The current study revealed that the most prevalent pathologies that indicated SNM include idiopathic bladder dysfunction, spinal cord injury, and $O A B$, which was diagnosed in $8(28.6 \%)$, $6(21.4 \%)$, and $4(14.3 \%)$ patients, respectively. A tertiary center experience in Riyadh, Saudi Arabia, reported the only indication for SNM as idiopathic urinary retention (10). Another study in Iraq reported slightly similar figures, with the idiopathic etiology as the most prevalent pathology, followed by neurological disease and spinal cord pathology (excluding complete spinal cord injuries) in $54.2 \%, 45.8 \%$, and $29.2 \%$, respectively (8).
Similar figures were reported in an Italian study, with idiopathic bladder dysfunction as the most frequent (11).

Regarding the success rate of SNM, the first stage success rate was $53.6 \%$, of which $42.9 \%$ had the device permanently implanted in the second stage. The rate is lower than the rates reported in the literature. Locally, a study done in Riyadh reported a higher success rate of $88 \%$ after the first stage implantation. Internationally, a Brazilian study reported similar findings with a success rate of $83.3 \%$ for the first stage implantation, and the pulse generator was implanted in the whole group (12). This high rate of success, compared to the present study, could be explained by several possibilities, such as insufficient pre-implantation sensitivity, disease severity and type, patient mentality, and socio-medical history. This is reflected in the Netherland study with a significant association between the late loss of therapeutic outcome and a history of former psychiatric complaints (13). None of the participants in both studies had lower urinary tract symptoms (LUTS) secondary to spinal cord injury, as an indication for SNM. However, studies done in Switzerland and Iraq included patients with spinal cord injuries and reported a first stage success rate of $67 \%$ and $70 \%$,

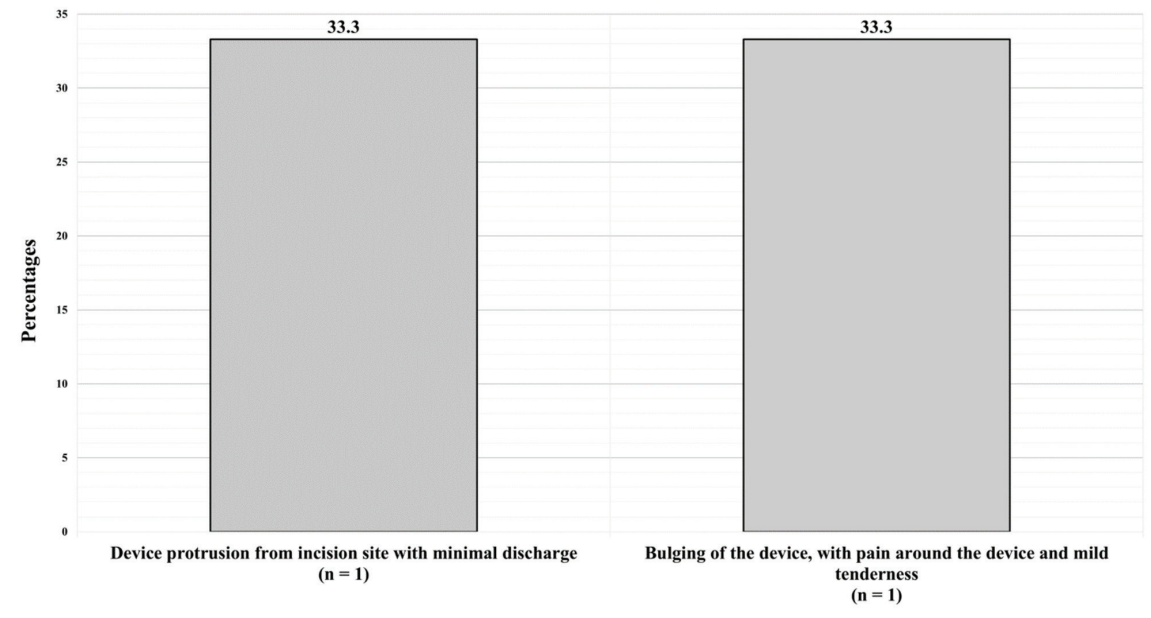

Figure 4. Complication rate among participants with device exchange

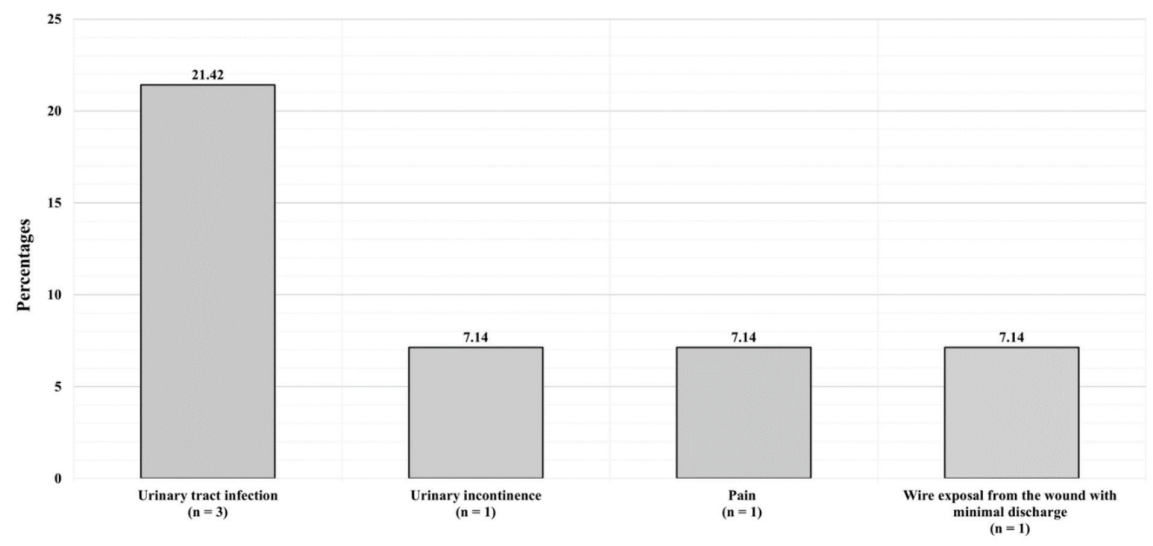

Figure 5. Complication rate among participants with electrode exchange/readjustment 


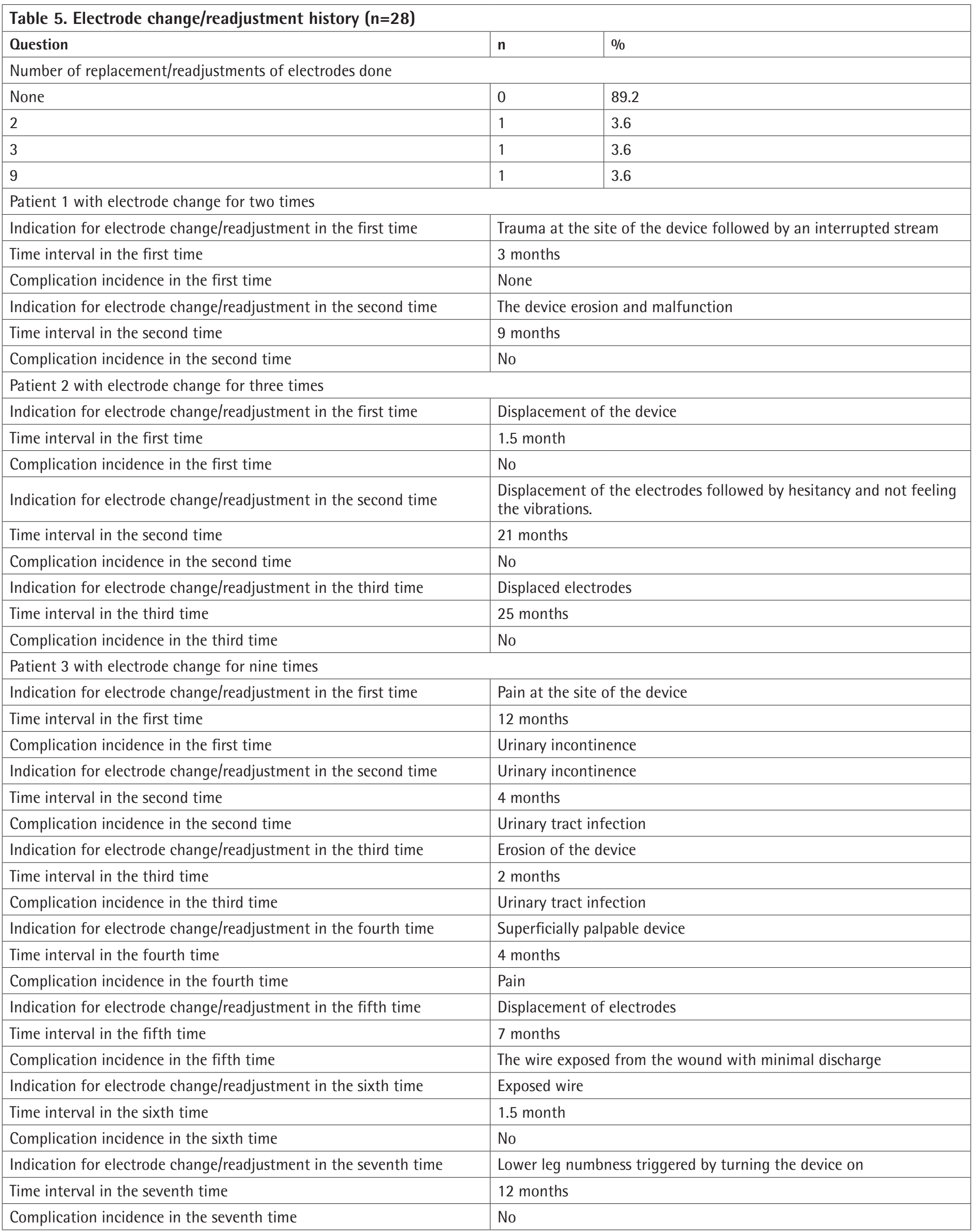




\begin{tabular}{|l|l|}
\hline Table 5. Continued \\
\hline Indication for electrode change/readjustment in the eighth time & Protruding device \\
\hline Time interval in the eighth time & 11 months \\
\hline Complication incidence in the eighth time & No \\
\hline Indication for electrode change/readjustment in the ninth time & Protruding device \\
\hline Time interval in the ninth time & 1.5 month \\
\hline Complication incidence in the ninth time & Urinary tract infection \\
\hline
\end{tabular}

respectively, which is still higher than the current study $(8,14)$. Lastly, the differences could be due to the sample size and SNM indications.

In terms of complications, the overall post-device implantation complication rate equated to $66.6 \%$ with device protrusion and dislocation as the most frequent complication. Similarly, a study in Riyadh reported a $70 \%$ rate of complications post-implantation, with box site infection and an undesirable sensation as the only complications (10). The Iraqi study also reported a similar rate $(62 \%)$ with infection and surgical site pain as the most frequent complications (8). Contrarily, a study done in Germany reported a lower post-device implantation complication rate $(31 \%)$, with lead migration as the most frequent (15). These discrepancies in the complication rates could be multifactorial, ranging from the nature of the practice in each center to patient factors (e.g., lifestyle, wound care, and pregnancy).

\section{Study Limitations}

A study limitation is that it did not include the progression of therapy that occurred during or after the study period, which may affect the overall patient outcome. Additionally, due to the retrospective nature of the current study, irregular attendance of scheduled visits could cause incomplete or lost data. Thus, the clinical implications of the current study should be interpreted with caution.

\section{Conclusion}

The observed complication rate was similar to literature reports, although the first stage success rate was lower than the reported rates both locally and internationally. This could be attributed to several reasons, such as the placebo effect of the first stage stimulation test, insufficient pre-implantation sensitivity, disease severity and type, patient mentality, and socio-medical history. In summary, based on the current study, SNM remains a safe and effective treatment option in carefully selected patients with LUTS, without any severe debilitating complications. With the increased use of SNM, regular patient evaluation and follow-up before SNM and after implantation are vital for future research. The current study could be a baseline and an incentive for larger multicenter studies. Exploring the outcomes of SNM on a larger scale will result in improved preoperative, perioperative, and postoperative patient care, thereby supporting a higher patient satisfaction rate and the alleviation of burdens both on the patients and the healthcare facility.

\section{Ethics}

Ethics Committee Approval: The Institutional Review Board of King Abdullah International Medical Research Center, the Ministry of National Guard-Health Affairs, Riyadh, Kingdom of Saudi Arabia, approved the study with the approval number NRC21R/095/03.

Informed Consent: Retrospective study.

Peer-review: Externally peer-reviewed.

\section{Authorship Contributions}

Concept: Mo.A., Y.G., M.A., R.A., Design: Mo.A., M.A., R.A., Data Collection or Processing: Y.G., Analysis or Interpretation: Mo.A., Literature Search: Y.G., Writing: M.A.

Conflict of Interest: No conflict of interest was declared by the authors.

Financial Disclosure: The authors declare that they have no relevant financial.

\section{References}

1. Wenzler DL, Burks FN, Cooney M, Peters KM. Proof of concept trial on changes in current perception threshold after sacral neuromodulation. Neuromodulation 2015;18:228-232.

2. Su X, Cutinella M, Agran JE, Dinsmoor DA. Comparison of Active Stimulating Electrodes of Sacral Neuromodulation. Neuromodulation 2017;20:799-806.

3. Ammirati $E$, Giammò $A$, Manassero $A$, Carone R. Neuromodulation in urology, state of the art. Urologia 2019;86:177-182.

4. Ghazwani YQ, Elkelini MS, Hassouna MM. Efficacy of sacral neuromodulation in treatment of bladder pain syndrome: long-term follow-up. Neurourol Urodyn 2011;30:1271-1275.

5. Anger JT, Cameron AP, Madison R, Saigal C, Clemens JQ; Urologic Diseases in America Project. Predictors of implantable pulse generator placement after sacral neuromodulation: who does better? Neuromodulation 2014;17:381384.

6. Meng L, Tian Z, Zhang W, Zhang Y, Wang J, Liao L, Ling Q, Zhang P, Wei $Z$, Zhong T, Xu Z, Wen W, Li J, Luo D. Influence of patient sex on the effectiveness of sacral neuromodulation: A cohort study from China. Int J Surg 2020;84:13-17. 
7. van Wunnik BP, Govaert B, Leong R, Nieman FH, Baeten CG. Patient experience and satisfaction with sacral neuromodulation: results of a single-center sample survey. Dis Colon Rectum 2011;54:95-100.

8. Al-Azzawi IS, Al-Tamimi MAJ. The first Iraqi experience in sacra neuromodulation for patients with lower urinary tract dysfunction. Arab J Urol 2018;16:391-396.

9. Shehab Z, Yaiesh S, Al-Terki A, Al-Shaiji T. Sacral neuromodulation: Report of initial experience in Kuwait. AJU 2018;16:16-17.

10. Mehmood S, Altaweel WM. Long-term outcome of sacral neuromodulation in patients with idiopathic nonobstructive urinary retention: Single-center experience. Urol Ann 2017;9:244-248.

11. Cardarelli S, D'Elia C, Cerruto MA, Curti P, Ostardo E, Signorello D, Pastorello $M$, Caleffi $G$, Molon A, Artibani W. Efficacy of sacral neuromodulation on urological diseases: a multicentric research project. Urologia 2012;79:9096.
12. Rios LA, Averbeck MA, Franca W, Sacomani CA, Almeida FG, Gomes CM. Initial experience with sacral neuromodulation for the treatment of lower urinary tract dysfunction in Brazil. Int Braz J Urol 2016;42:312-320.

13. Weil EH, Ruiz-Cerdá JL, Eerdmans PH, Janknegt RA, Van Kerrebroeck PE. Clinical results of sacral neuromodulation for chronic voiding dysfunction using unilateral sacral foramen electrodes. World J Urol1998;16:313-321.

14. Wöllner J, Krebs J, Pannek J. Sacral neuromodulation in patients with neurogenic lower urinary tract dysfunction. Spinal Cord 2016;54:137-140.

15. Kavvadias T, Huebner M, Brucker SY, Reisenauer C. Management of devicerelated complications after sacral neuromodulation for lower urinary tract disorders in women: a single center experience. Arch Gynecol Obstet 2017;295:951-957. 